\title{
Closed-loop compensation of dielectric charge induced by ionizing radiation
}

\author{
Manuel Dominguez-Pumar ${ }^{1}$, Sergi Gorreta ${ }^{1}$, Joan Pons-Nin ${ }^{1}$, Faustino Gomez-Rodriguez ${ }^{2}$, Diego M \\ Gonzalez-Castaño $^{2}$, Michele Muschitiello ${ }^{3}$ \\ ${ }^{1}$ Micro and Nano Technologies Group, DEE, Universitat Politècnica de Catalunya, Barcelona, Spain. ${ }^{2}$ Radiation Physics Laboratory, \\ Universidade de Santiago de Compostela, Spain. ${ }^{3}$ European Space Agency, ESTEC, Noordwijk, The Netherlands.
}

\begin{abstract}
This work investigates the capability of dielectric charge control loops to cope with charge induced by ionizing radiation. To this effect, a MEMS variable capacitor has been irradiated with X-Rays and Gamma-radiation in three scenarios: without polarization, using an open-loop dielectric charge mitigation strategy and using a closed-loop control method. The results show that charge effects induced by radiation can be partially compensated using dielectric charge control.
\end{abstract}

Index Terms-Dielectric charging control, MEMS reliability, radiation effects, ionizing radiation.

\section{INTRODUCTION}

Operation of MEMS devices is often hampered by dielectric charging phenomena. Closed-loop methods to control the amount of actuation-induced net dielectric charge have been demonstrated in [1]-[4]. Ionizing radiation can also induce dielectric charge in MEMS devices operating in space or harsh environments [5]-[7]. The purpose of this work is to study whether these closed-loop control methods can effectively cope with charge induced by ionizing radiation. To this effect several experiments have been performed on which MEMS devices are irradiated with X-ray and gamma radiation while a dielectric charge control is being implemented.

The sigma-delta control used in this work is a sampledtime circuit that allows setting a desired level of net dielectric charge $Q_{\text {target }}$ [4]. During each sampling period, $T_{S}$, either a BIT0 or a BIT1 waveform is applied to the device, see Fig. 1. In the contactless case, these waveforms produce complementary effects on the charge dynamics: BIT0s increase the amount of net dielectric charge $Q$, and thus increase the voltage shift of the $C$ - $V$ curve, $V_{s h}$, whereas BIT1s decrease both $Q$, and $V_{s h}$. The decision about which symbol to apply at each sampling period is made with an indirect charge measurement: the difference between two capacitance measurements obtained during each symbol at two bipolar actuation voltages, $\Delta C=C\left(V^{+}\right)-C\left(V^{-}\right)$, see Fig. 1 . Under some conditions of the $C-V$ curve [3] (for example, a parabolic characteristic), it is possible to discern whether the net charge is below or above the desired target level by comparing $\Delta C$ with a threshold value.

In a first order control of charge, the decision on whether to apply BIT0 or BIT1 is made based on the instantaneous measurement of the total dielectric charge (obtained through $\Delta C$ ). If this value is below the desired level of charge, a BIT0 is applied to increase it. If it is above, a BIT1 will be applied instead. This means that the control is always trying to compensate any deviations from the desired target charge value
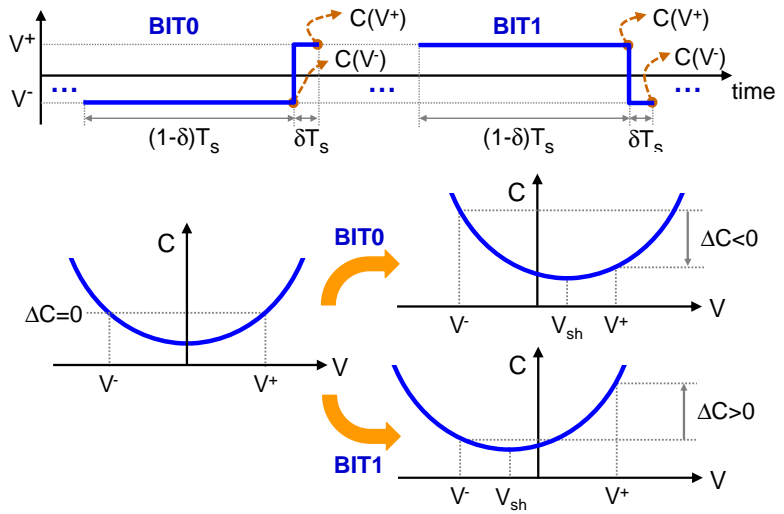

Fig. 1. Top: actuation waveforms, or symbols BIT0 and BIT1. For each symbol, capacitance measurements made at times $(1-\delta) T_{S}$ and $T_{S}$ allow to obtain $\Delta C$ as $C\left(V^{+}\right)-C\left(V^{-}\right)$. Bottom: example illustrating the complementary effect of applying each symbol on the voltage shift $V_{s h}$, thus on the net charge $Q$, and on $\Delta C$. Applying only BIT0s increases $V_{s h}$ and decreases $\Delta C$, whereas BIT1s decrease $V_{s h}$ and increase $\Delta C$.

by generating an adequate sequence of actuation symbols. In presence of charge induced by radiation, the control scheme will have to change the actuation sequence so as to take into account this charge generation. Globally, there is a balance between the charge being continuously leaked out of the dielectric, that being injected by the control actuation and that being generated by ionizing radiation.

The charge sensing part of the scheme must only be able to distinguish between the events: net charge above or below the desired target value. In this work, a second order sigma-delta control loop (see Fig. 2) has been used in order to avoid the possible presence of plateaus, which imply a local lack of control [4]. The appropriate time scale for the

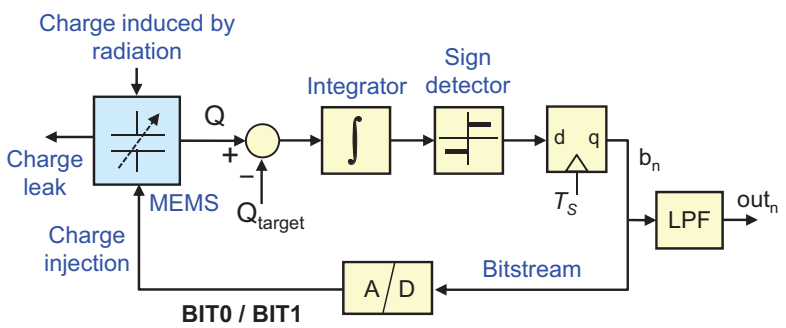

Fig. 2. Block diagram of the charge controller. The bitstream $b_{n}$ is averaged to obtain the real-time information signal out ${ }_{n}$. Although the sensing signals are capacitances, equivalent charges are used here for more clarity. 

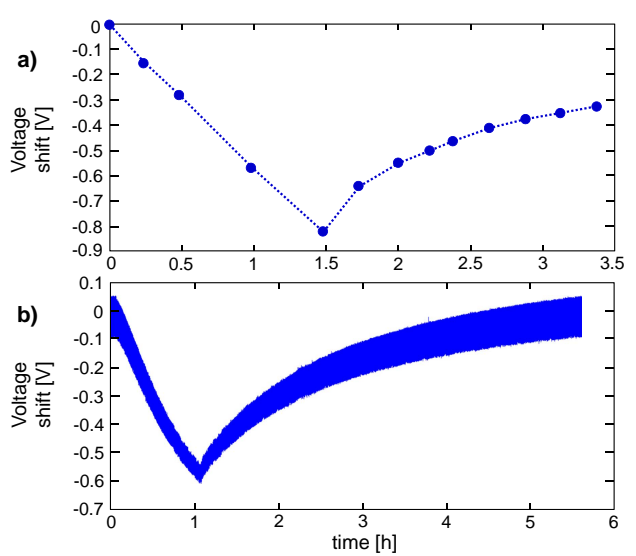

Fig. 3. (a) Evolution of $V_{s h}$ when the MEMS is first X-Ray irradiated with $D R=1.3 \mathrm{~Gy} / \mathrm{s}$ for $1.5 \mathrm{~h}$, then not irradiated for the next $2 \mathrm{~h}$. The actuation voltage was $V=0$ during all this time. (b) Evolution of $V_{s h}$ when alternate BIT0 and BIT1 symbols are applied to the MEMS. The device is initially discharged, then X-Ray irradiated with $D R=1.3 \mathrm{~Gy} / \mathrm{s}$ for $1 \mathrm{~h}$. Symbol parameters: $V^{+}=-V^{-}=10 \mathrm{~V}, \delta=1 / 3, T_{S}=1.2 \mathrm{~s}$.

sampling frequency is determined by the dielectric charging time constants, usually very large (hours or days). In this work sampling times of the order of 1-2 seconds are used.

As in [3], [4], the control was implemented with a precision impedance meter. The MEMS, also used in [3], is a parallelplate structure fabricated with PolyMUMPS technology. The top-moveable plate is a stack of $1.5 \mu \mathrm{m}$ polysilicon and $0.5 \mu \mathrm{m}$ gold layers of $360 \times 360 \mu \mathrm{m}^{2}$ area. The bottom plate is the doped $\mathrm{Si}$ substrate, with a $0.6 \mu \mathrm{m} \mathrm{Si}_{3} \mathrm{~N}_{4}$ layer deposited on top of it. The substrate contact is made through a bias hole in the $\mathrm{Si}_{3} \mathrm{~N}_{4}$ layer. The air gap between the top plate and the $\mathrm{Si}_{3} \mathrm{~N}_{4}$ is $2.75 \mu \mathrm{m}$ and the pull-in voltage is $24 \mathrm{~V}$.

\section{X-RAY AND GAMma RADIATION EXPERIMENTS}

The X-Ray experiments were performed at the Radiation Physics Laboratory of University of Santiago de Compostela. The die containing the DUT was placed on an uncovered 84-pin package facing the radiation source. The DUT was irradiated by a $50 \mathrm{kV}$ X-Ray beam delivered by an Oxford Instruments Neptune tube without filtration. The beam output was characterized by measuring the spectrum and the exposure rate. Two dose rates $(D R)$ were delivered to the $\mathrm{Si}_{3} \mathrm{~N}_{4}$ layer: $1.3 \mathrm{~Gy} / \mathrm{s}$ a and $0.65 \mathrm{~Gy} / \mathrm{s}$. The dose rates used in the experiments are generally high. In the case of the gamma radiation, they are within the standard dose rate window $(0.01$ to $0.1 \mathrm{~Gy} / \mathrm{s}$ ) allowed for accelerated test according to the ESCC 22900 basic specification.

The first test investigated whether radiation induces noticeable dielectric charging. The DUT, initially discharged, was irradiated with a $D R=0.65 \mathrm{~Gy} / \mathrm{s}$ for $1.5 \mathrm{~h}$ and not irradiated for the next $2 \mathrm{~h}$. To ensure that no charge was induced by mechanisms other than radiation, the voltage between the MEMS electrodes/plates was set to zero. Short-range $C-V$ measurements provided the evolution of $V_{s h}$. The results, plotted in Fig. 3(a), clearly show an increasing amount of negative charge during irradiation. When radiation ceases, the zero bias tends to remove the charge. However, such charge removal is slow and only partial at the end of the experiment.

The effect of using an open-loop mitigation strategy for actuation-induced charge was also investigated. A zeroaverage sequence of alternate BIT0 and BIT1 symbols actuated

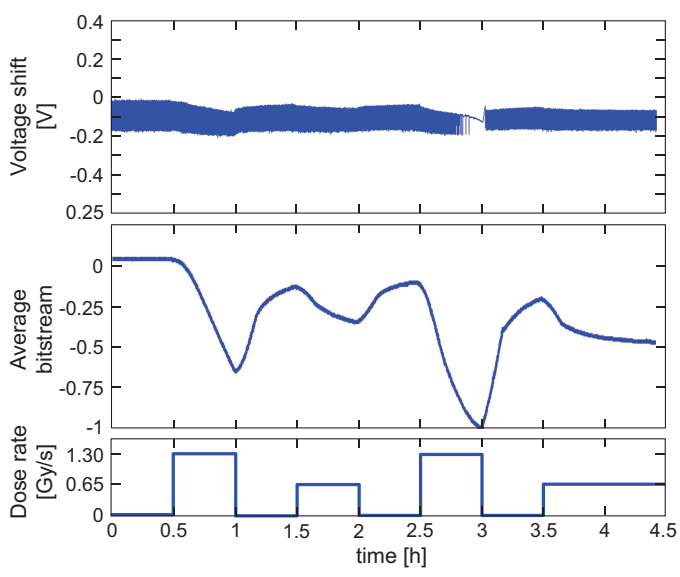

Fig. 4. Evolution of $V_{s h}$ and of the average bitstream when the control method is applied to the MEMS to obtain $V_{s h}=0$ (zero net charge). $V^{+}=-V^{-}=10 \mathrm{~V}, \delta=1 / 3, T_{S}=1.2$ s. Bottom: dose rates delivered. At $\mathrm{t}=3 \mathrm{~h}$ the control goes into saturation for a short time.

the DUT during a second experiment, which included $D R=1.3$ Gy/s irradiation for $1 \mathrm{~h}$. The evolution of $V_{s h}$, Fig. 3(b), indicates an increase of negative charge during the irradiation phase. After irradiation, the DUT slowly returns to the initial state.

Finally, a closed-loop control was used to compensate the radiation effects seen in the previous experiments. The loop was configured to set $V_{s h}=0 \mathrm{~V}$ (zero net charge), while a burstlike series of irradiation steps was applied to the DUT, see Fig. 4. The figure shows that $V_{s h}$ is kept around zero during all the experiment, thus demonstrating the effectiveness of the control to partially compensate the charge induced by radiation. Furthermore, the average bitstream clearly correlates with the sequence of dose rates applied. Irradiation phases increase the amount of negative charge to be compensated by the control, thus the average bitstream tends to -1 (BIT0 or $V^{-}$dominant). In the rest phases the average tends to zero, indicating that net charge is being removed.

Fig. 4 also shows that $30 \mathrm{~min}$ of rest phase is not time enough to completely discharge the DUT after $D R=1.3 \mathrm{~Gy} / \mathrm{s}$ irradiation. Note also that a saturation episode occurs just before $\mathrm{t}=3 \mathrm{~h}$ : only BIT0s are applied and the control is at this point no longer able to compensate all the radiation-induced charge. As expected, non-saturated control is reached again sometime after irradiation ceases.

The next set of experiments was performed applying lower dose rates. In this case, the sensitivity of the control loop was increased by using lower actuation voltages, which induce less actuation-generated charge at each symbol. In Fig. 5, control with $V^{+}=-V^{-}=7 \mathrm{~V}$ was applied to set $V_{s h}=0$, while the DUT was exposed to $0.675 \mathrm{~Gy} / \mathrm{s}$ and $0.3375 \mathrm{~Gy} / \mathrm{s}$ irradiation steps, followed by zero radiation. The results demonstrate that $V_{s h}$ is fairly kept around zero. The behaviour of the average bitstream clearly correlates with the irradiation sequence.

Next, the target was set to $V_{s h}=0.5 \mathrm{~V}$, while the DUT was under a $D R=0.675 \mathrm{~Gy} / \mathrm{s}$ for $75 \mathrm{~min}$, preceded and succeeded by $1 \mathrm{~h}$ rest intervals. Fig. 6(a) shows that this non-zero target charge is successfully achieved after the first 20 minutes.

The effect of non-symmetrical voltages was also investigated. In Fig. 6(b), control with $V^{+}=8 \mathrm{~V}$ and $V^{-}=-6 \mathrm{~V}$ and the same target $V_{s h}=0.5 \mathrm{~V}$ was used. After $50 \mathrm{~min}$ of rest phase, 
$0.675 \mathrm{~Gy} / \mathrm{s}$ and $0.3375 \mathrm{~Gy} / \mathrm{s}$ irradiation steps were applied. In the rest phase $V_{s h}$ is kept around the target value, with an average bitstream of -0.65 , a value noticeably below that obtained with $V^{+}=-V^{-}=7 \mathrm{~V}$, see Fig. 6(a). This is an expected result, since the new values of $V^{-}$and $V^{+}$imply that each BIT0 injects less positive charge, then more BIT0s must be applied to reach $V_{s h}=0.5 \mathrm{~V}$. As in Fig. 4, saturation is seen sometime after irradiation starts: applying these "weak" BIT0s is not enough to compensate the negative charge induced by radiation and the actuation. However, non-saturated control is retaken after irradiation decreases to $D R=0.3375 \mathrm{~Gy} / \mathrm{s}$.

An experiment for investigating the effect of applying the charge control method under Gamma radiation was performed at the ESA-ESTEC ${ }^{60} \mathrm{Co}$ facility. The ${ }^{60} \mathrm{Co}$ source, with $60 \mathrm{TBq}$ activity, is housed in a Gammabeam $\AA$ 150-C irradiator. With the source raised to the irradiation position, the gamma beam produced by the ${ }^{60} \mathrm{Co}$ decay irradiated the DUT placed opposite to the irradiator window. The facility dosimetry is based on a 2670 Farmer electrometer equipped with a Farmer Type $25710.6 \mathrm{cc}$ air ionisation chamber, with calibration under an ISO 17025 accreditation scheme.

In the experiment, charge control with target $V_{s h}=0 \mathrm{~V}$ was continuously applied to the DUT, under successive steps with no radiation for $15 h$, irradiation with $D R=0.051 \mathrm{~Gy} / \mathrm{s}$ for $37 \mathrm{~h}$ and, again, no radiation for $35 \mathrm{~h}$. The results, see Fig. 7, demonstrate that $V_{s h}$ is kept around zero during the experiment. Furthermore, a clear correlation exists between the average bitstream and the irradiation sequence.

\section{CONCLUSIONS}

In experiments involving either X-Ray or Gamma radiation, the time evolution of $V_{s h}$ and the correlation between the control bitstream and the dose rates applied indicate that net charge induced by radiation can be compensated. Real-time information about the charge being induced by radiation is obtained by monitoring the average bitstream. This may allow to use this technique in radiation detection applications. To the knowledge of the authors, this is the first time that a successful partial compensation of radiation-induced effects using active dielectric charge control techniques is shown.

Acknowledgements: The authors wish to thank ESA-ESTEC for letting them use their ${ }^{60} \mathrm{Co}$ facility and, in particular, the kind support from Dr. Nicolas Saillen. This work was

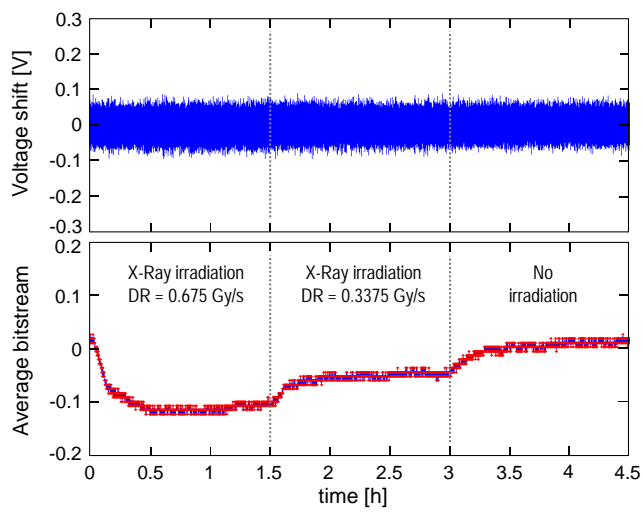

Fig. 5. Evolution of $V_{s h}$ and of the average bitstream when control is applied to obtain $V_{s h}=0$ (zero charge). Two different dose rates are delivered to the DUT. $V^{+}=-V^{-}=7 \mathrm{~V}, \delta=1 / 4, T_{S}=1.6 \mathrm{~s}$.
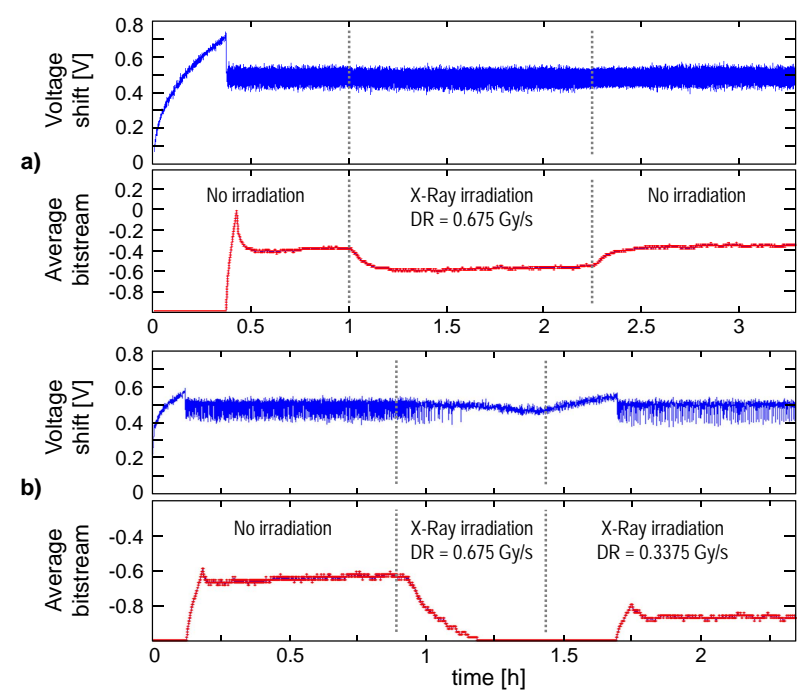

Fig. 6. Evolution of $V_{s h}$ and of the average bitstream when control is applied to obtain $V_{s h}=0.5 \mathrm{~V}$. Case (a): $V^{+}=-V^{-}=7 \mathrm{~V}, \delta=1 / 4, T_{S}=1.6$ $\mathrm{s}$ and $D R=0.675 \mathrm{~Gy} / \mathrm{s}$ delivered to the DUT, preceded and succeeded by zero radiation steps. Case (b): $V^{+}=8 \mathrm{~V}, V^{-}=-6 \mathrm{~V}, \delta=1 / 4, T_{S}=1.6 \mathrm{~s}$ and two different dose rates delivered to the DUT after a zero radiation phase; at $\mathrm{t}=110$ min the control goes into saturation.

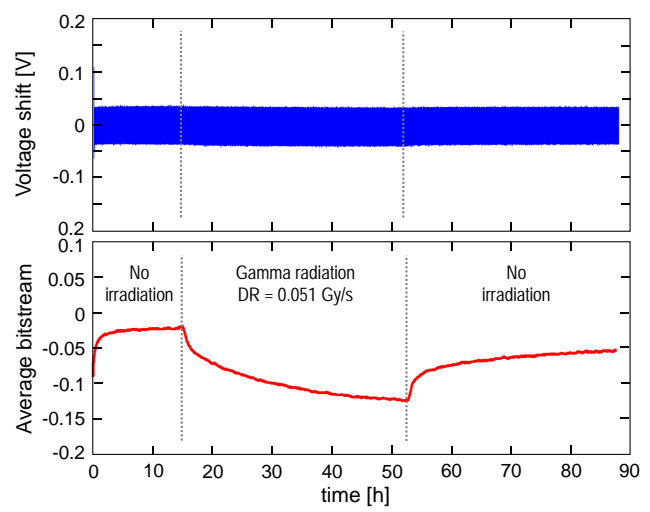

Fig. 7. Evolution of $V_{s h}$ and of the average bitstream when control is applied to obtain $V_{s h}=0 . D R=0.051 \mathrm{~Gy} / \mathrm{s}$ is delivered to the DUT, preceded and succeeded by zero radiation steps. $V^{+}=-V^{-}=5 \mathrm{~V}, \delta=1 / 3, T_{S}=1.2 \mathrm{~s}$.

supported in part by the Spanish Ministry MINECO under Project TEC2013-48102-C2-1-P.

\section{REFERENCES}

[1] M. Dominguez, D. Lopez, D. Molinero, and J. Pons, "Dielectric charging control for electrostatic MEMS switches," in SPIE Conf. on Defense, Security and Sensing, vol. 7679, pp. 1-11, Orlando, 2010.

[2] E. Blokhina, S. Gorreta, D. Lopez, D. Molinero, O. Feely, J. Pons-Nin, and M. Dominguez, "Dielectric charge control in electrostatic MEMS positioners / varactors," IEEE-JMEMS, vol. 21, pp. 559-573, 2012.

[3] S. Gorreta, J. Pons, E. Blokhina, O. Feely, and M. Domínguez, "Deltasigma control of dielectric charge for contactless capacitive MEMS," IEEE-JMEMS, vol. 23, no. 4, pp. 829-841, 2014.

[4] S. Gorreta, J. Pons, E. Blokhina, and M. Domínguez, "A second order delta-sigma control of dielectric charge for contactless capacitive MEMS," in press in IEEE-JMEMS, 2015.

[5] V. Theonas, M. Exarchos, G. Papaioannou, and G. Konstantinidis, "RF MEMS dielectric sensitivity to electromagnetic radiation," Sensors and Actuators A: Physical, vol. 132, no. 1, pp. 25 - 33, 2006.

[6] H. Shea, "Radiation sensitivity of microelectromechanical system devices," Journal of Micro/Nanolithography, MEMS, and MOEMS, vol. 8, no. 3, p. 031303, 2009.

[7] A. Hartzell, M. Da Silva, and H. Shea, "In-use failures," in MEMS Reliability, ser. MEMS Reference Shelf. Springer US, 2011, pp. 85-177. 